\title{
Car-following Characteristics of Adaptive Cruise Control from Empirical Data
}

\author{
Noah J. Goodall ${ }^{1}$ and Chien-Lun Lan ${ }^{2}$ \\ Pre-print version. Final version in Journal of Transportation Engineering, Part A: Systems, 146 (9), Sep. 2020. \\ https://doi.org/10.1061/JTEPBS.0000427
}

\begin{abstract}
Computer-driven vehicles will behave differently than human-driven vehicles due to changes in perception abilities, precision control, and reaction times. These changes are expected to have profound impacts on capacity, yet few models of automated driving are based on empirical measurements of computer-driven vehicles in real traffic. To this end, this paper investigates characteristics of an early form of longitudinal control automation, a commercially available adaptive cruise control (ACC) system driven in real traffic. Two car-following models were calibrated to a vehicle with ACC. First, the Intelligent Driver Model was reformulated to comply with ACC design standards then calibrated to match speed and range data from the test vehicle. The vehicle with ACC was found to decelerate less severely than predicted by the model when tested in severe braking and unimpeded acceleration scenarios. Second, the Wiedemann 99 model was calibrated, as it is the default car-following model in the traffic microsimulation software program VISSIM and can therefore be implemented cheaply and quickly in sophisticated models of roadways worldwide. Four parameters of the Wiedemann 99 model were measured directly from field observations of the test vehicle: standstill distance, startup time, unimpeded acceleration profile, and maximum desired deceleration. Simulation results in VISSIM were found to match the adaptive cruise control in unimpeded acceleration tests. These findings will benefit researchers and modelers seeking more accurate models of car-following behavior with adaptive cruise control and automated longitudinal control.
\end{abstract}

\footnotetext{
${ }^{1}$ Senior Research Scientist, Virginia Transportation Research Council, 530 Edgemont Road, Charlottesville, VA 22903. Email: noah.goodall@vdot.virginia.gov. ORCiD: 0000-0002-3576-9886.

${ }^{2}$ Research Scientist, Virginia Transportation Research Council, 530 Edgemont Road, Charlottesville, VA 22903. Email: chien-lun.lan@vdot.virginia.gov. ORCiD: 0000-0002-1760-6135.
} 


\section{INTRODUCTION}

The act of driving is becoming increasingly automated. In the United States, 29 states and the District of Columbia have enacted some form of automated vehicle legislation (National Conference of State Legislatures 2019), while five automotive manufacturers and two technology companies anticipate having full-speed automated vehicles that will not require invehicle driver monitoring by 2021 (Lewis and Grossman 2019). To prepare for automated vehicles, governments have begun updating planning and capacity models to account for differences between human-driven and computer-driven vehicles. A recent study by the Virginia Transportation Research Council found that while planning models for automated vehicles were highly dependent on estimates of how automation will affect capacity, the existing literature provides no clear estimate on capacity changes due to increasing automation (Miller and Kang 2019). Existing methods to estimate road capacity are based on the driving behavior of humans, and computer-driven vehicles may behave quite differently. For example, the rubber-necking effect where drivers pause to observe a shoulder crash may be significantly reduced with automated vehicles.

Adaptive cruise control (ACC) can be described as an intermediate stage of vehicle automation. Adaptive cruise control uses radar, lidar, or stereo camera sensors to track the distance to the vehicle ahead and attempts to maintain a consistent time headway to the lead vehicle (Weinberger et al. 2001). Adaptive cruise control is now offered as a feature on many new vehicles from most major automakers, and 7.2\% of new vehicles sold in 2020 are expected to be equipped with ACC technologies (Wayland 2015).

The driving behavior of an automated vehicle can be described using several different parameters, including reaction time, acceleration profile, deceleration profile, emergency deceleration rate, following distance, etc. Specific aspects of automated vehicle behavior, including adaptive cruise control behavior, are generally not patented nor disclosed by their developers, and are instead handled as trade secrets (Milanés and Shladover 2014). As the carfollowing logic employed in adaptive cruise control systems are likely to be used in more advanced automated vehicles, there is a value in studying and understanding the car-following behavior of various adaptive cruise control systems.

Adaptive cruise control systems developed by different manufacturers often use different settings and may exhibit a range of behaviors. More car-following models based on field observations of production vehicles with ACC are needed to improve the ability to model their behavior and understand their impact on traffic flow and capacity. The purpose of this research is to establish a car-following model that reflects the behavior of a production vehicle with adaptive cruise control, and to provide guidance for modeling a vehicle with adaptive cruise control using commercial microscopic simulation software.

This research makes several novel contributions. Two car-following models were calibrated to match the longitudinal behavior of a 2017 Audi Q7 with ACC driven on public roads in traffic. Two models were selected: the Intelligent Driver Model (IDM) and the Wiedemann 99 model. 
The original IDM (Treiber et al. 2000) was enhanced in 2010 to minimize hard braking events due to its collision-free assumptions (Kesting et al. 2010), and this enhanced IDM was selected for this analysis for several reasons. Unlike many other models designed for human drivers, the enhanced IDM was developed specifically to model ACC systems (Kesting et al. 2010). Four of its parameters can be directly-measured from a single test vehicle: minimum time gap, gap at stopped traffic, maximum acceleration, and desired deceleration. Because maximum acceleration, desired deceleration, and minimum time gap are separate parameters, the enhanced IDM can be calibrated to adhere to ISO standards for ACC vehicles (ISO 2010). Finally, IDM has been used many times to estimate capacity (Kesting et al. 2007b; a, 2010; Milanés and Shladover 2014; Shladover et al. 2012) and model ACC vehicles from empirical data (Milanés and Shladover 2014; Su et al. 2016); by using IDM, this effort allows direct comparisons with previous work. This paper represents only the second attempt to calibrate the IDM based on a vehicle with ACC and the first with an Audi Q7.

Several parameters of the Wiedemann 99 model used in the microscopic traffic simulation software VISSIM were derived directly from field measurements of the same 2017 Audi Q7 with ACC. The Wiedemann 99 model is psychophysical designed around human perception and reaction abilities, and is therefore not ideal for modeling a computer-controlled system. A different or custom model might be preferred, but modelers and transportation agencies may be hesitant to implement an unfamiliar model. The main motivation for using Wiedemann 99 is its widespread use by government transportation agencies and contractors through the simulation software package VISSIM. VISSIM is the most widely used microscopic traffic simulation software according to a survey conducted by Brackstone et al. (2012). Governments have made significant investments in developing highly sophisticated and calibrated VISSIM models of many roadways. By calibrating the Wiedemann 99 model to empirical car-following, this effort builds on traffic modelers' investment and training to provide improved model results that can be implicated quickly at minimal cost. This is the approach taken by the European Union's CoEXist project. The Federal Highway Administration also supports this as a viable option for modeling automated vehicle behavior (Mahmassani et al. 2018). This paper represents the first effort to measure Wiedemann 99 parameters for a named vehicle with ACC using disclosed empirical data collected on public roads.

\section{LITERATURE REVIEW}

There have been several attempts to model the behavior of vehicles with adaptive cruise control. Brackstone and McDonald (1999) reviewed the history of car-following models, while Xiao and Gao (2010) provided an overview of ACC systems. Jerath and Brennan (2012) used the General Motors car-following model to estimate capacities with ACC-enabled vehicles, and found drastically higher capacities as market penetration neared 100 percent. Ntousakis et al. (2015) used the Gipps car-following model (Gipps 1981) as implemented in the AIMSUM microscopic simulation software to model automated vehicle behavior to estimate capacities at various ACC penetration rates and found capacity improvements when ACC time headways were set to $1 \mathrm{~s}$ or 
less. VanderWerf et al. (2002) used Monte Carlo simulations of a ACC car-following model (VanderWerf et al. 2001) with 1.4 s desired headways to estimate capacity impacts. Cellular automata models of ACC behavior have been developed by Yuan et al. (2009). Recently, Makridis et al (2020) measured the response time of an actual ACC-controller as 0.8-1.2 s, similar to human response time.

Two studies have attempted to model adaptive cruise control systems based on empirical data from production vehicles. In 2014, Milanés and Shladover (2014) measured the behavior of four Nissan Infiniti M56s equipped with ACC following another vehicle following pre-planned accelerations on a test track. The IDM was fit to the empirical data but was found to have an undershoot problem with lower than expected accelerations and a time gap error. The authors developed a simplified car-following model based on distance and speed errors between the ACC vehicle and the leading vehicle. The new model was able to predict the accelerations of the test vehicle with greater accuracy than the IDM.

In 2016, Su et al. (2016) studied a 2013 Cadillac SRX equipped with ACC on freeways and collected speeds and gaps from the vehicle's own sensors. The authors were unable to successfully fit the IDM to their data, and instead developed a three-phase car-following model based on leading, transitioning, and following modes. Their results showed good agreement between IDM and empirical data for speeds and acceleration but noted that small speed errors compounded to produce large gap errors, with root-mean-square errors as high as 69 meters. At the test speeds of $30 \mathrm{~m} / \mathrm{s}$, this translated into gap errors of 2 seconds or more, nearly double the 1.1 second headways used by the vehicle with ACC. In calibrating the IDM, the authors set the desired speed at $39.5 \mathrm{~m} / \mathrm{s}(88 \mathrm{mi} / \mathrm{h})$ in order to produce realistic behavior, requiring the vehicle to stay behind a slower lead vehicle through simulation to prevent speeding.

Most of the studies in the literature have used assumptions of adaptive cruise control characteristics when modeling vehicle behavior or estimating capacity. Of the two studies which used empirical data (Milanés and Shladover 2014; Su et al. 2016), both operated the vehicles at speeds of over $25 \mathrm{~m} / \mathrm{s}(55 \mathrm{mi} / \mathrm{h})$ and did not investigate characteristics of ACC systems at low speeds. This study contributes to the literature through an analysis of a previously untested manufacturer and model of vehicle with adaptive cruise control, as well as unexamined behaviors at low speeds.

\section{INTELLIGENT DRIVER MODEL}

Most adaptive cruise control systems studied in the literature have been best described by the Intelligent Driver Model (IDM). A commonly used IDM is an enhanced version developed by Kesting et al. (2010) which defines an acceleration target from the following equation:

$$
a_{I D M}=a\left[1-\left(\frac{v}{v_{0}}\right)^{\delta}-\left(\frac{s_{0}+\max \left[0, v T+\frac{v \Delta v}{2 \sqrt{a b}}\right]}{s}\right)^{2}\right]
$$




$$
\begin{aligned}
& v=\text { current vehicle speed } \\
& v_{0}=\text { desired speed } \\
& s=\text { gap } \\
& s_{0}=\text { gap at stopped traffic } \\
& T=\text { minimum time gap } \\
& a=\text { maximum acceleration } \\
& b=\text { desired deceleration, expressed as a positive value } \\
& \delta=\text { free acceleration exponent }
\end{aligned}
$$

To our knowledge, this equation does not appear in the literature in this form. Kesting et al. (2010) neglected to include the maximum function on the last term to prevent it from taking negative values. This was updated on the IDM website and reflected by Milanés and Shladover (2014). In the equation displayed by Milanés and Shladover (2014), however, the last term was not raised to second power as it was by Kesting et al. (2010) and others (Li et al. 2015; Liebner et al. 2012; Su et al. 2016). This error has been corrected in Equation 1.

Most production vehicles with ACC adhere to the ISO standard 15622 on adaptive cruise control performance requirements (ISO 2010). The standard states that "The average rate of change of automatic deceleration (negative jerk) shall not exceed $2.5 \mathrm{~m} / \mathrm{s}^{3}$ (average over $1 \mathrm{~s}$ )" (ISO 2010). The IDM equation can be modified to adhere to the standard.

$$
\begin{gathered}
a_{I D M}=\left\{\begin{array}{cl}
j_{\max }+a_{t-r}, & a_{I D M}-a_{t-r}<j_{\max } \\
a_{I D M}, & \text { otherwise }
\end{array}\right. \\
j_{\max }=\text { maximum jerk over one second }\left(\mathrm{m} / \mathrm{s}^{3}\right) \\
t=\text { current timestep } \\
r=\text { simulation time steps per second }
\end{gathered}
$$

ISO 15622 further mandates a maximum deceleration rate: "The average automatic deceleration of ACC systems shall not exceed $3.5 \mathrm{~m} / \mathrm{s}^{2}$ (average over $2 \mathrm{~s}$ )" (ISO 2010). The IDM equation is amended to adhere to the standard.

$$
\begin{gathered}
a_{I D M}=\left\{\begin{array}{cl}
2 r b_{\max }-\sum_{i=t-1}^{t+1-2 r} a_{i}, & \frac{1}{2 r}\left(a_{I D M}+\sum_{i=t-1}^{t+1-2 r} a_{i}\right)<b_{\max } \\
a_{I D M}, & \text { otherwise }
\end{array}\right. \\
b_{\max }=\begin{array}{c}
\text { maximum deceleration over } 2 \mathrm{~s}, \text { expressed as a } \\
\text { negative value }\left(\mathrm{m} / \mathrm{s}^{2}\right)
\end{array}
\end{gathered}
$$

Combining both amended equations yields the following: 


$$
a_{I D M}=\left\{\begin{array}{cl}
j_{\max }+a_{t-r}, & a_{I D M}-a_{t-r}<j_{\max } \\
2 r b_{\max }-\sum_{i=t-1}^{t+1-2 r} a_{i}, & \frac{1}{2 r}\left(a_{I D M}+\sum_{i=t-1}^{t+1-2 r} a_{i}\right)<b_{\max } \\
a_{I D M}, & \text { otherwise }
\end{array}\right.
$$

In the same paper, Kesting et al. (2010) acknowledge that, as a collision-free model, the IDM leads to excessive braking when following a leading vehicle as it assumes the lead vehicle may brake at its maximum rate at any moment. To relax this assumption and produce more realistic driving, they introduce the constant acceleration heuristic $(\mathrm{CAH})$ which assumes leading vehicles will maintain current speeds for a few seconds. The formula for acceleration using the $\mathrm{CAH}$ is given by:

$$
\begin{gathered}
a_{C A H}=\left\{\begin{array}{c}
\frac{v^{2} \tilde{a}_{l}}{v_{l}^{2}-2 s \tilde{a}_{l}}, \quad v_{l}\left(v-v_{l}\right) \leq-2 s \tilde{a}_{l} \\
\tilde{a}_{l}-\frac{\left(v-v_{l}\right)^{2} \Theta\left(v-v_{l}\right)}{2 s}, \quad \text { otherwise }
\end{array}\right. \\
v_{l}=\text { current speed of leading vehicle }(m / s) \\
\tilde{a}_{l}=\min \left(a_{l}, a\right) \\
\Theta, \text { Heaviside step function: } H(n)= \begin{cases}0, & n<0 \\
1, & \mathrm{n} \geq 0\end{cases}
\end{gathered}
$$

According to Kesting et al. (2010), the CAH accelerations are too relaxed in some situations, showing zero acceleration when moderate braking is practical. If the IDM produces extreme decelerations while the $\mathrm{CAH}$ recommends accelerations greater than the desired deceleration $b$, then the situation can be described as mildly critical and the acceleration of a vehicle with ACC is equal to the comfortable deceleration plus a fraction $1-c$ of the IDM deceleration. The constant $c$ refers to a coolness factor, which corresponds to the sensitivity of the ACC vehicle to the preceding vehicle. Kesting et al. (2010) use $c=0.99$, and recommend realistic values between 0.95 and 1.00. The formula for their enhanced IDM model for ACC vehicles is given by:

$$
\begin{gathered}
a_{A C C}=\left\{\begin{aligned}
a_{I D M}, & a_{I D M} \geq a_{C A H} \\
(1-c) a_{I D M}+c\left[a_{C A H}+b \tanh \left(\frac{a_{I D M}-a_{C A H}}{b}\right)\right], & \text { otherwise }
\end{aligned}\right. \\
b=\text { desired deceleration, expressed as a positive value } \\
\left(\mathrm{m} / \mathrm{s}^{2}\right) \\
c=\text { coolness factor }
\end{gathered}
$$


Often $a_{A C C}$ will be greater than zero and produce abnormally high accelerations. A final check should confirm that $a_{I D M}$ should be used if $a_{A C C}$ results in a positive value.

$$
a_{A C C}= \begin{cases}a_{I D M}, & a_{A C C} \geq 0 \\ a_{A C C}, & \text { otherwise }\end{cases}
$$

This model, $a_{A C C}$, is referred to as the ACC model throughout this paper, while the earlier version without the constant acceleration heuristic, $a_{I D M}$, is referred to as the IDM model.

The formulation of IDM in equations 1-4 is unique to this paper, and not found in existing literature. The formulation of the ACC model in equations 5-7, however, are reproduced directly from Kesting et al. (2010).”

\section{DATA COLLECTION}

The test vehicle is a 2017 Audi Q7 with adaptive cruise control functionality. In order to develop a car-following model, at least two points are required: speed difference between the leading and following vehicle, and the gap between the leading and following vehicle. By measuring the rate of change of these characteristics, one can determine the accelerations of both leading and following vehicles. These data were obtained in live traffic by using a smart phone's GPS sensor placed inside the vehicle to determine speed once per second, from which acceleration was calculated. The gap to the leading vehicle was obtained by a laser scanner mounted on a tripod inside the Audi measuring the rear of the leading vehicle. The laser scanner provided the gap between vehicles, while the speed difference was obtained by calculating the rate of change of the gap and comparing it to the speed of the Audi as measured by the GPS. The laser scanner installation is shown in Figure 1.

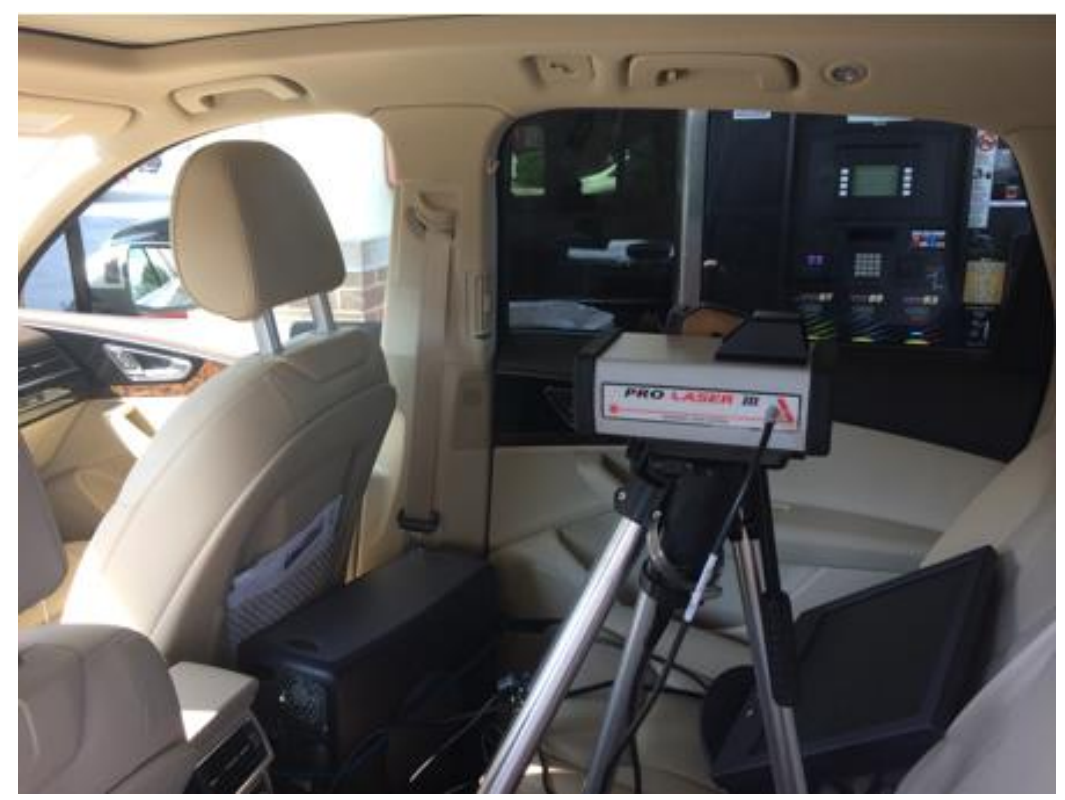

Fig. 1 Laser scanner installed in the test vehicle. 
The test vehicle has five time headway settings of 1, 1.3, 1.8, 2.4, and 3.6 seconds. Testing was conducted using the 1.8 second headway, which is the default setting whenever the vehicle's power is cycled. The test vehicle was driven in live traffic on signalized arterials and highways in order to maximize the potential for lead vehicle decelerations. Finally, the vehicle was tested with ACC with a desired speed of $96 \mathrm{~km} / \mathrm{h}(60 \mathrm{mi} / \mathrm{h})$ from a standing start with no leading vehicles present to determine its unimpeded acceleration profile.

Data collection was filmed with dash-mounted digital video cameras that were synced to the GPS time. These cameras not only show traffic conditions and presence of a leading vehicle, they also monitor the vehicle dashboard and record vehicle status, ACC settings, and ACC engagement, as well as unusual traffic scenarios such as cut-in maneuvers that quickly shorten the vehicle's perceived following distance. A screenshot of the timestamped video is shown in Figure 2.

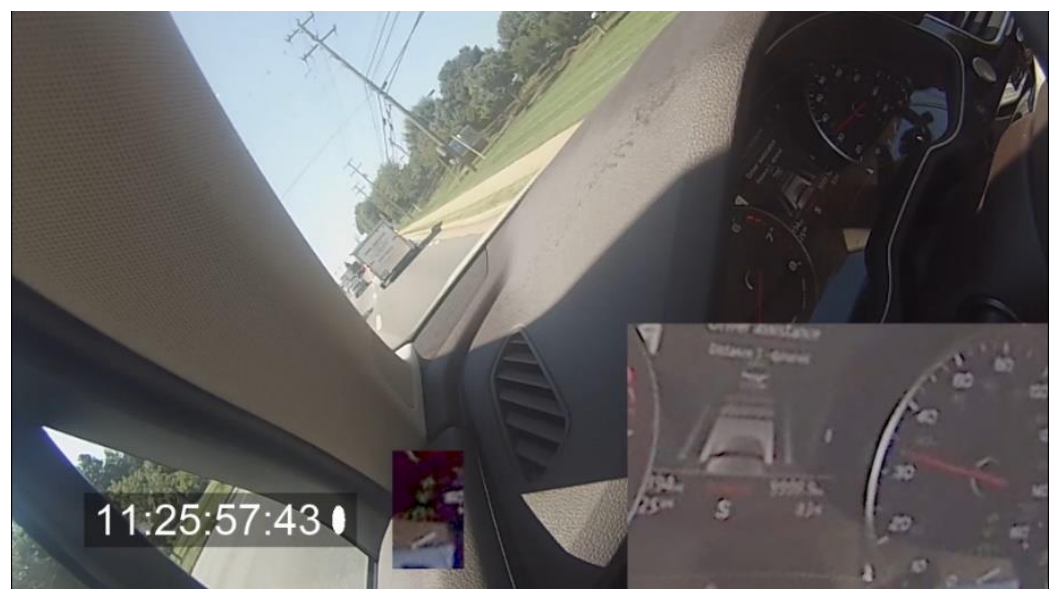

Fig. 2 Consolidated timestamped video of test vehicle with ACC engaged.

Data from the laser scanner and smartphone GPS sensor application were fused into a master file with a common time stamp. As all sensors record data at different intervals, some intermediate readings had to be extrapolated from sensor readings. For example, GPS records position once per second, while gap was recorded approximately three times per second. Linear interpolation was used to extrapolate gaps, positions, and speeds at 0.1 second intervals for consistency. Noisy range data was checked against the video file to determine if the position of the lead vehicle was responsible or if the lidar sensor had targeted the wrong object. Faulty range measurements were removed, and data was smoothed using linear interpolation where practical.

\section{ACC CHARACTERISTICS RELEVANT TO CAR-FOLLOWING}

Four characteristics of car-following common to many models were studied as part of this effort: standstill distance, startup time, acceleration profile, and deceleration profile. 


\section{Standstill Distance}

Standstill distance is the desired gap between the front of the following vehicle and the back of the leading vehicle when both vehicles are stationary. The IDM refers to this distance as jam distance (Kesting et al. 2010).

Eight instances of vehicles stopped traffic were recorded while the vehicle was operating with ACC. In five instances, the gap was measured at 3.65 meters, with the remaining measurements between 3.35 and 4.2 meters. Some measurements may disagree somewhat with actual bumper-to-bumper distance, as the range finder was aimed at the broadest surface, often the back of the vehicle, and may have measured slightly farther than the rear-most part of the lead vehicle's bumper. A standstill distance of 3.5 meters is the most practical value and was used in calibration of the IDM.

\section{Startup Time and Distance}

A critical component of intersection capacity and queue clearance formulas is the time from when a lead vehicle accelerates from stopped to when the following vehicle begins to accelerate. In traffic engineering, this time gap is used to estimate the capacity of a traffic signal, generally using a default value of two seconds (Transportation Research Board 2010).

Startup time was measured from timestamped video recorded while the vehicle was in a stopped queue at a traffic signal and operating with ACC. Two times were measured: (a) the time between when the brake light of the lead vehicle turned off and the ACC vehicle began moving, and (b) the time between when the lead vehicle appeared to move and when the ACC vehicle began to move. While eight samples are reported for standstill distance, only six were recorded for startup time and distance because in two samples ACC was disengaged after the vehicle stopped. The results are shown in Table 1. The time between both vehicles moving ranged between 1.2 and 3.0 seconds, with an average value of 1.73 seconds. This is similar to the 2 second default value used in traffic engineering.

Table 1. Startup Times and Distances for Queued Vehicle using ACC

\begin{tabular}{llll}
\hline Sample & $\begin{array}{l}\text { Time between lead brake } \\
\text { light off and ACC vehicle } \\
\text { movement }(s)\end{array}$ & $\begin{array}{l}\text { Time between lead vehicle } \\
\text { movement and ACC vehicle } \\
\text { movement }(s)\end{array}$ & $\begin{array}{l}\text { Distance traveled by lead vehicle } \\
\text { distance before ACC vehicle } \\
\text { movement }(m)\end{array}$ \\
\hline A & 2.84 & 2.02 & 1.0 \\
B & 2.68 & 1.94 & 3.0 \\
C & 2.36 & 1.44 & 1.5 \\
D & 2.70 & 1.64 & 2.5 \\
E & 2.27 & 1.24 & 1.2 \\
F & 2.02 & 1.27 & 1.2 \\
\hline Average & 2.48 & 1.59 & 1.73 \\
\hline
\end{tabular}

\section{Acceleration}

Car-following models use acceleration rates as inputs, often based on a vehicle's maximum comfortable acceleration when unimpeded. To determine acceleration rates, the test vehicle's 
ACC was engaged with a desired speed of $96 \mathrm{~km} / \mathrm{h}(60 \mathrm{mi} / \mathrm{h})$ from standstill while accelerations were derived from locations measurements taken once per second from GPS. The results of nine test runs were averaged to produce an acceleration profile at different speeds, shown in Table 2. Accelerations at times exceed the ISO 15622 standard's maximum of $2.0 \mathrm{~m} / \mathrm{s}^{2}$ (ISO 2010).

Table 2. Average ACC Acceleration from Stationary to $96 \mathrm{~km} / \mathrm{h}(60 \mathrm{mi} / \mathrm{h})$

\begin{tabular}{ll}
\hline $\begin{array}{l}\text { Vehicle } \\
\text { Speed } \\
(\mathrm{km} / \mathrm{h})\end{array}$ & $\begin{array}{l}\text { Average } \\
\text { Acceleration } \\
\left(\mathrm{m} / \mathrm{s}^{2}\right)\end{array}$ \\
\hline 0 & 0.36 \\
10 & 2.02 \\
20 & 2.10 \\
30 & 2.66 \\
40 & 2.36 \\
50 & 2.01 \\
60 & 1.75 \\
70 & 1.53 \\
80 & 1.17 \\
90 & 0.57 \\
\hline
\end{tabular}

\section{Deceleration}

Car-following models often use desired and maximum decelerations as inputs. For an ACC vehicle, maximum deceleration may be defined as the maximum allowable under ISO 15622 , defined as $-3.5 \mathrm{~m} / \mathrm{s}^{2}$ over two seconds. The desired deceleration is less severe, and values of -2 $\mathrm{m} / \mathrm{s}^{2}$ is used by both Kesting et al. (2010) and Milanés and Shladover (2014). In observations, the $98 \%$ of the test vehicle's decelerations were less severe than $-2 \mathrm{~m} / \mathrm{s}^{2}$ as seen in Figure 3 . Therefore, a value of $-2 \mathrm{~m} / \mathrm{s}^{2}$ was selected as the desired deceleration, although it must be expressed as a positive value when used in the IDM. 


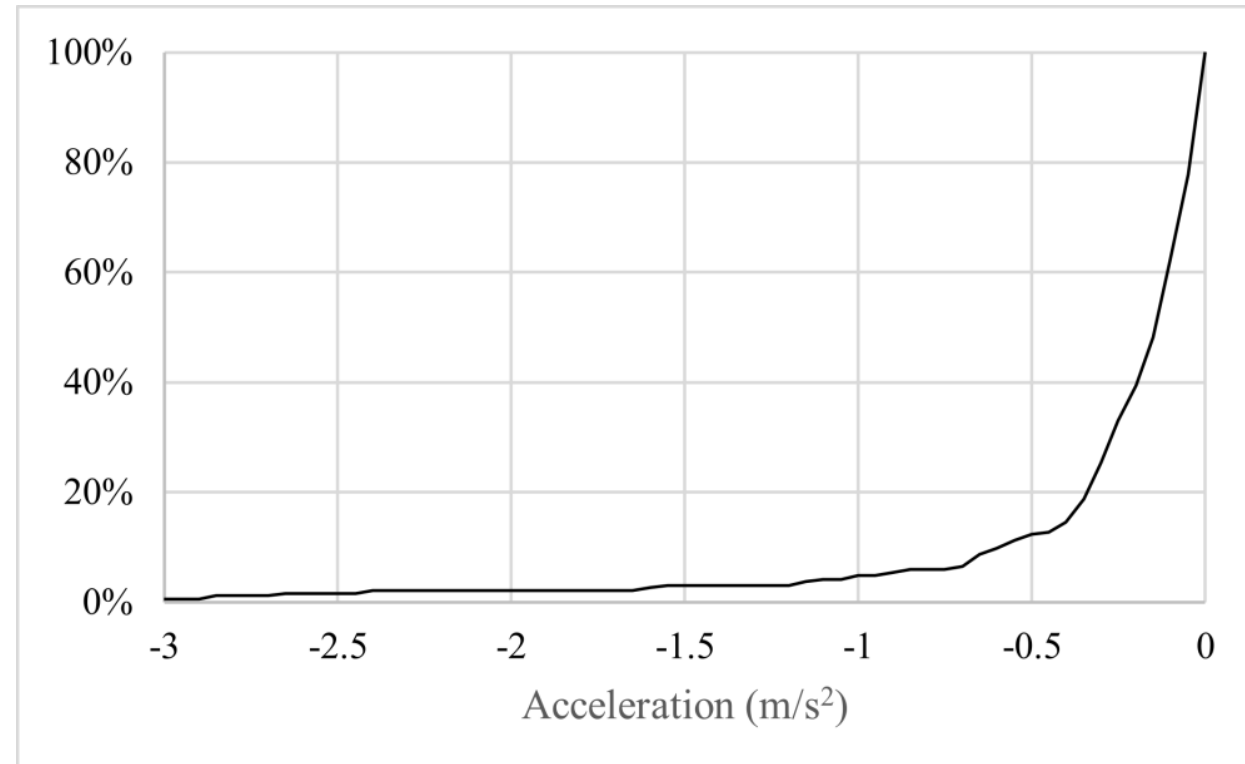

Fig. 3. Empirical distribution function of test vehicle decelerations with ACC engaged.

\section{IDM AND ACC MODEL DEVELOPMENT}

ACC behavior attributes studied in the previous section were used to determine parameters for the IDM and ACC models developed by Kesting et al. (2010). The parameters are shown in Table 3, with sources for selected values listed.

Table 3. IDM and ACC Model Parameters

\begin{tabular}{llll}
\hline Parameter & Symbol & Value & Source \\
\hline Desired speed & $v_{0}$ & Varies & Data \\
Free acceleration exponent & $\delta$ & 4 & (Kesting et al. 2010; Milanés and Shladover 2014) \\
Desired time gap & $T$ & $1.8 \mathrm{~s}$ & (Audi AG 2016) \\
Jam distance & $s_{0}$ & $3.5 \mathrm{~m}$ & Data \\
Maximum acceleration & $a$ & $2.0 \mathrm{~m} / \mathrm{s}^{2}$ & Data, (ISO 2010) \\
Desired deceleration & $b$ & $2.0 \mathrm{~m} / \mathrm{s}^{2}$ & Data, (Kesting et al. 2010; Milanés and Shladover \\
& & & 2014) \\
Coolness factor & $c$ & 0.99 & (Kesting et al. 2010) \\
Maximum deceleration over 2 s & $b_{\max }$ & $-3.5 \mathrm{~m} / \mathrm{s}^{2}$ & (ISO 2010) \\
Maximum jerk over 1 & $j_{\max }$ & $-2.5 \mathrm{~m} / \mathrm{s}^{3}$ & (ISO 2010) \\
\hline
\end{tabular}

The model was tested on a 30-second data set with the test vehicle decelerating from 22.2 $\mathrm{m} / \mathrm{s}(50 \mathrm{mi} / \mathrm{h})$ to $5 \mathrm{~m} / \mathrm{s}(11 \mathrm{mi} / \mathrm{h})$ to avoid a car slowed in a queue, then accelerating to $17.7 \mathrm{~m} / \mathrm{s}$ $(40 \mathrm{mi} / \mathrm{h})$ while following the lead vehicle. This is the largest speed oscillation tested in any attempt to model real-world ACC behavior. The IDM and the ACC models result in overly cautious braking, decelerating at $-4 \mathrm{~m} / \mathrm{s}^{2}$ when time gaps were just under 3 seconds, while the 
test vehicle allowed time gaps to reach 1.3 seconds before braking. The speed and position of the vehicles over time is shown in Figures 4 and 5.

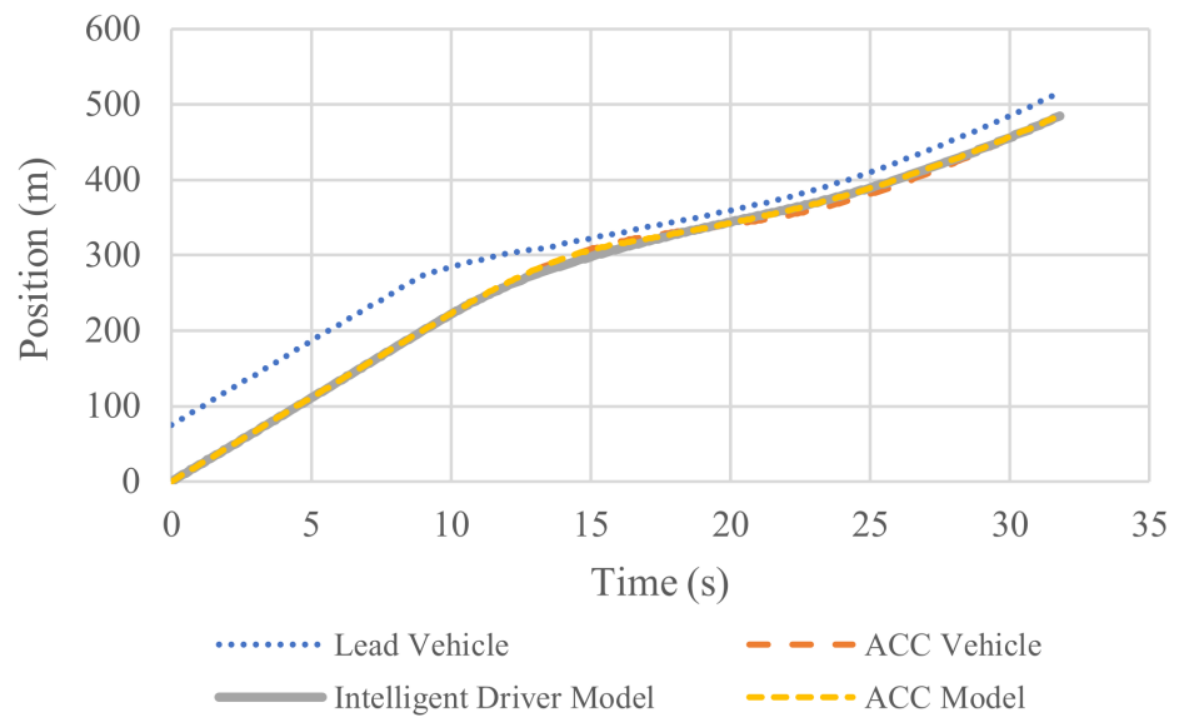

Fig. 4. Position vs. time for vehicles and models in the test scenario.

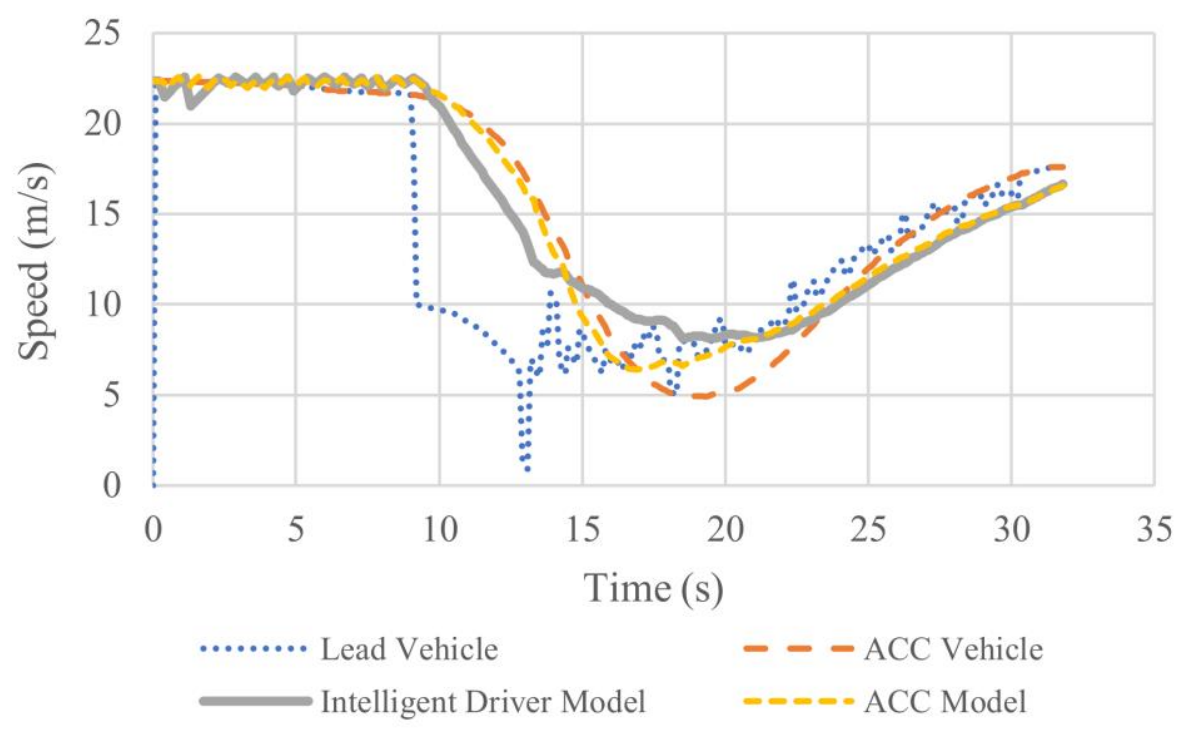

Fig. 5. Speed vs. time for vehicles and models in the test scenario.

Most factors cannot be calibrated to improve the performance in the test scenario without worsening performance in acceleration tests. Increasing the free acceleration threshold $\delta$, for example, produces a delayed deceleration similar to the test vehicle, but produces nearly constant acceleration from standstill of $2.0 \mathrm{~m} / \mathrm{s}^{2}$ until desired speed is attained, which contradicts the field data collected in Table 2. 


\section{VISSIM SETTINGS}

VISSIM is a widely used microscopic traffic simulation software package developed by PTV AG (2018). The software uses two car-following models when simulating vehicles. The first, Wiedemann 74, is based on published research conducted on the German Autobahn in the early 1970s (Wiedemann 1974) and updated in 1992 (Wiedemann and Reiter 1992). The second, Wiedemann 99, has not been published but appears similar to Wiedemann 74 in terminology, e.g. parameters $\mathrm{CC} 0$ in Wiedemann 99 and $a x$ in Wiedemann 74 both refer to standstill distance. Both models recognize thresholds between different states or "regimes" of driving such as free, following, closing, and emergency (PTV AG 2018). Olstam and Tapani (2004) provided a thorough analysis of the Wiedemann car-following logic and default settings.

Because Wiedemann 99 has 10 adjustable parameters compared to just three with Wiedemann 74, PTV Group recommends using Wiedemann 99 for modeling automated vehicles such as those with adaptive cruise control (Sukennik 2018).

Two examples in the literature have attempted to provide example Wiedemann 99 parameters that might apply to automated vehicles or vehicles with adaptive cruise control. Sukennik (2018) of PTV Group developed values for each of the ten Wiedemann 99 parameters for a cautious, normal, and all-knowing automated vehicle. The models are included in VISSIM version 11 as pre-installed driving behavior models. The exact values used in the VISSIM models are reproduced in Table 4. Bierstedt et al. (2014) also developed example values for Wiedemann 99 parameters to model the effect of ACC on freeway capacity. Their values are based on assumptions of ACC behavior such as faster and more precise responses to preceding vehicle accelerations. The proposed parameter values are reproduced in Table 4.

Table 4. Recommended Wiedemann 99 Settings for AVs and ACC from the Literature

\begin{tabular}{|c|c|c|c|c|c|c|c|}
\hline \multirow[b]{2}{*}{ Parameter } & \multirow[b]{2}{*}{ Units } & \multicolumn{3}{|c|}{ AVs (Sukennik 2018) } & \multicolumn{3}{|c|}{ ACC (Bierstedt et al. 2014) } \\
\hline & & Cautious & Normal & All-knowing & Aggressive & Intermediate & Conservative \\
\hline $\mathrm{CC} 0$ & $\mathrm{~m}$ & 1.5 & 1.5 & 1.0 & 1.0 & 1.25 & 1.5 \\
\hline $\mathrm{CC} 1$ & $\mathrm{~s}$ & 1.5 & 0.9 & 0.6 & 0.5 & 0.8 & 1.2 \\
\hline $\mathrm{CC} 2$ & $\mathrm{~m}$ & 0.0 & 0.0 & 0.0 & 2.0 & 3.0 & 4.0 \\
\hline $\mathrm{CC} 3$ & $\mathrm{~s}$ & -10.0 & -8.0 & -6.0 & -8.0 & -12.0 & -16.0 \\
\hline $\mathrm{CC} 4$ & $\mathrm{~m} / \mathrm{s}$ & -0.1 & -0.1 & -0.1 & -0.1 & -0.35 & -0.6 \\
\hline CC5 & $\mathrm{m} / \mathrm{s}$ & 0.1 & 0.1 & 0.1 & 0.1 & 0.35 & 0.6 \\
\hline CC6 & $1 /(\mathrm{m} \cdot \mathrm{s})$ & 0.0 & 0.0 & 0.0 & 0.0 & 0.0 & 0.0 \\
\hline CC7 & $\mathrm{m} / \mathrm{s}^{2}$ & 0.1 & 0.1 & 0.1 & 0.4 & 0.25 & 0.1 \\
\hline CC8 & $\mathrm{m} / \mathrm{s}^{2}$ & 3.0 & 3.5 & 4.0 & 0.4 & 3.5 & 3.0 \\
\hline CC9 & $\mathrm{m} / \mathrm{s}^{2}$ & 1.2 & 1.5 & 2.0 & 2.0 & 1.5 & 1.0 \\
\hline
\end{tabular}

Based on the findings of this research and the assumptions and values from the literature, values for the Wiedemann 99 model parameters are proposed in Table 5 for use in VISSIM software. Ideally, parameters would be calibrated to match traffic conditions. In the absence of a 
data set of many interacting vehicles using ACC, some parameters were calibrated to a single vehicle analyzed as part of this study. For parameters without supporting empirical data, values from Bierstedt et al. (2014) and Sukennik (2018) were used. When these sources disagreed, the most recent reference (Sukennik 2018) was used. In addition to the parameters, the car-following model should use deterministic values when possible, which can be implemented by un-checking the "Use implicit stochastics" box within the Driving Behavior editing window in VISSIM 11. The maximum and desired acceleration profiles should be set to match the results of the ACC acceleration tests in Table 2. Finally, maximum and minimum accelerations at each speed should be set equivalent to the average value taken from Table 2, unless the model is designed to reflect a range of acceleration profiles from different manufacturers and vehicle models.

A comparison of acceleration profiles observed from the ACC test vehicle, as predicted by the IDM and ACC models (which are identical during unimpeded acceleration), and from a VISSIM simulation using the settings in Table 5 are shown in Figure 6. Accelerations match well, with all vehicles reaching $25 \mathrm{mi} / \mathrm{h}$ within 37 meters of each other.

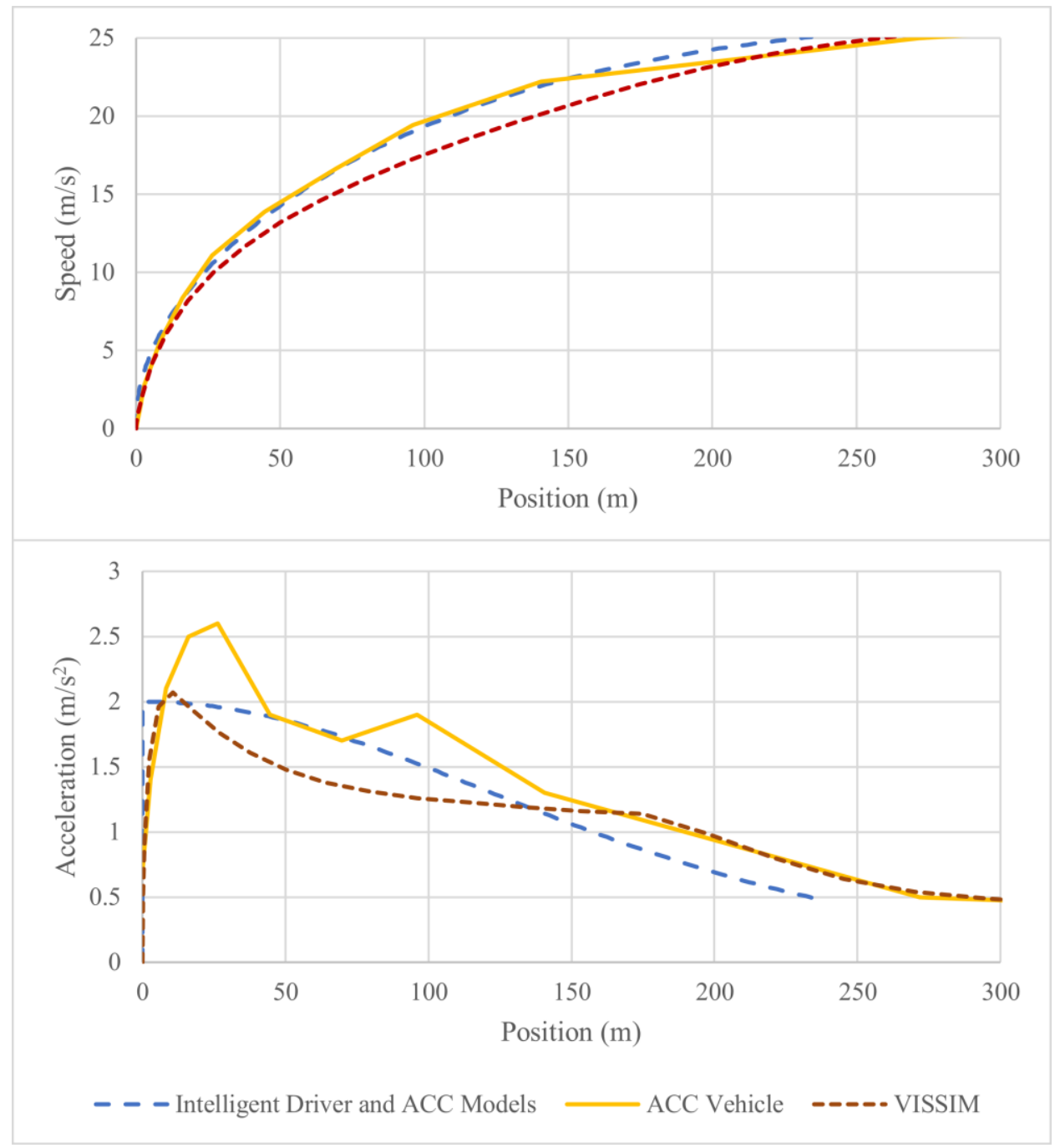

Fig. 6. Accelerations and speeds of unimpeded acceleration. 
Table 5. VISSIM Wiedemann 99 Model Recommended Parameters for Vehicle with ACC

\begin{tabular}{|c|c|c|}
\hline $\begin{array}{l}\text { Wiedemann } \\
99 \text { Parameter }\end{array}$ & Description & $\begin{array}{l}\text { Recommended } \\
\text { Value }\end{array}$ \\
\hline $\mathrm{CC} 0$ & $\begin{array}{l}\text { Standstill distance: The desired gap between two stationary } \\
\text { vehicles. Observed distance was } 3.5 \mathrm{~m} \text {. }\end{array}$ & $3.5 \mathrm{~m}$ \\
\hline $\mathrm{CC} 1$ & $\begin{array}{l}\text { Following distance: The minimum desired time gap between } \\
\text { two vehicles. The vehicle's default following distance was } \\
1.8 \text { s, although this should be altered based on known or } \\
\text { estimated settings. }\end{array}$ & $1.8 \mathrm{~s}$ \\
\hline $\mathrm{CC} 2$ & $\begin{array}{l}\text { Longitudinal oscillation: The gap distance beyond the } \\
\text { minimum safety distance at which a vehicle will accelerate to a } \\
\text { leading vehicle. Test vehicle set at } 1.8 \text { s following distance } \\
\text { would accelerate when following distance exceeded } 1.9 \text { s at } \\
\text { speeds of } 15-20 \mathrm{~m} / \mathrm{s} \text {, resulting in a value of } 1.5 \text { to } 2.0 \text { meters. }\end{array}$ & $2.0 \mathrm{~m}$ \\
\hline $\mathrm{CC} 3$ & $\begin{array}{l}\text { Perception threshold for following: The number of seconds } \\
\text { prior to which reaching a safety distance at which deceleration } \\
\text { begins, expressed as a negative value. Value recommended in } \\
\text { the literature (Bierstedt et al. 2014; Sukennik 2018). }\end{array}$ & $-8.0 \mathrm{~s}$ \\
\hline $\mathrm{CC} 4$ & $\begin{array}{l}\text { Negative speed difference: Threshold for negative difference } \\
\text { in speed between leading and following vehicle for reaction } \\
\text { during the following regime. Value recommended by Sukennik } \\
\text { (2018). }\end{array}$ & $-0.1 \mathrm{~m} / \mathrm{s}$ \\
\hline $\mathrm{CC} 5$ & $\begin{array}{l}\text { Positive speed difference: Threshold for positive difference in } \\
\text { speed between leading and following vehicle for reaction } \\
\text { during the following regime. Value recommended by Sukennik } \\
\text { (2018). }\end{array}$ & $0.1 \mathrm{~m} / \mathrm{s}$ \\
\hline CC6 & $\begin{array}{l}\text { Influence speed on oscillation: Measure of the impact of gap } \\
\text { on speed oscillation. Value recommended in the literature } \\
\text { (Bierstedt et al. 2014; Sukennik 2018). }\end{array}$ & $0 /(\mathrm{m} \cdot \mathrm{s})$ \\
\hline $\mathrm{CC} 7$ & $\begin{array}{l}\text { Oscillation during acceleration: Limits the jerk during the } \\
\text { first time step while a vehicle is in the free regime. Value from } \\
\text { observations of the test vehicle's initial acceleration when } \\
\text { starting from standstill with no leading vehicle. }\end{array}$ & $0.36 \mathrm{~m} / \mathrm{s}^{2}$ \\
\hline $\mathrm{CC} 8$ & $\begin{array}{l}\text { Desired acceleration starting from standstill: Value from } \\
\text { maximum allowable acceleration in ISO } 15622 \text { (ISO 2010). }\end{array}$ & $2.00 \mathrm{~m} / \mathrm{s}^{2}$ \\
\hline CC9 & $\begin{array}{l}\text { Desired acceleration at } 80 \mathrm{~km} / \mathrm{h} \text { : Value from acceleration } \\
\text { tests. }\end{array}$ & $1.17 \mathrm{~m} / \mathrm{s}^{2}$ \\
\hline
\end{tabular}




\section{CONCLUSIONS}

Governments must adapt their planning and capacity models to the increase in computercontrolled driving, yet the car-following models which serve as the basis for capacity models are based on human drivers and limited by human visual perception and psychology. Adaptive cruise control, where a vehicle follows a leading vehicle at a constant time headway, is available on production vehicles and may exhibit similar car-following behavior to prototype highly automated vehicles. The purpose of this study was to investigate the characteristics of a production vehicle with adaptive cruise control which may apply to modeling efforts.

A 2017 Audi Q7 with adaptive cruise control was equipped with a GPS sensor, rangefinder, and video to determine the vehicle's speed, acceleration, gap distance to the proceeding vehicle, and ACC status. Data was consolidated under a common time stamp and analyzed for various car-following characteristics.

Four attributes were measured directly: standstill distance $(3.5 \mathrm{~m})$, startup time $(1.59 \mathrm{~s})$, accelerations (Table 2), and decelerations (effective maximum of $2.0 \mathrm{~m} / \mathrm{s}^{2}$ ). The empirical data was compared to the Intelligent Driver Model over a 30-second scenario exhibiting braking from $22.5 \mathrm{~m} / \mathrm{s}$ to $5 \mathrm{~m} / \mathrm{s}$, the most drastic deceleration of an ACC vehicle analyzed in the car-following literature. The ACC vehicle showed less severe deceleration when encountering congestion, allowing headways of 1.3 seconds before decelerating compared to IDM's 3 second headways. Finally, sample values for parameters of the Wiedemann 99 car-following model were provided based on the empirical data and the literature so that ACC vehicles can be modeled in the widely used microscopic simulation software package VISSIM with less reliance on assumed behavior of ACC and automated vehicles.

Future research could involve validating the ACC car-following models developed by Milanés and Shladover (2014) and Su et al. (2016) against the data collected in this project, as the vehicle used here was produced by a different manufacturer and may exhibit different carfollowing behavior. Additionally, a scenario could be staged in such a way that it could be recreated in VISSIM to test the performance of the recommended Wiedemann 99 parameters in a realistic car-following situation. Finally, the effect of lane changing and lateral movements on ACC behavior should be investigated.

\section{DATA AVAILABILITY STATEMENT}

Some or all data, models, or code that support the findings of this study are available from the corresponding author upon reasonable request. Available data include speed/acceleration/gap measurements, VISSIM trajectories and models, and IDM calculations.

Some or all data, models, or code generated or used during the study are proprietary or confidential in nature and may only be provided with restrictions (e.g. anonymized data). In-car video may be shared in short, anonymized sections to protect identities of other road users. 


\section{ACKNOWLEDGEMENTS}

This work was sponsored by the Virginia Department of Transportation. The authors thank Eun (Tina) Lee for her assistance with data collection.

\section{REFERENCES}

Audi AG. 2016. Owner's manual, 2017 Q7. Audi AG, Ingolstadt, Germany.

Bierstedt, J., A. Gooze, C. Gray, J. Peterman, L. Raykin, and J. Walters. 2014. Effects of nextgeneration vehicles on travel demand and highway capacity. FP Think Working Group.

Brackstone, M., and M. McDonald. 1999. "Car-following: a historical review." Transp. Res. Part F: Traff. Psych. and Behaviour, 2(4), 181-196.

Brackstone, M., Montanino, M., Daamen, W., Buisson, C., and Punzo, V. 2012. "Use, calibration, and validation of traffic simulation models in practice: results of Web-based survey." 91st Annual Meeting of the Transp. Res. Board, Washington, DC.

Gipps, P. G. 1981. "A behavioural car-following model for computer simulation.” Transp. Res. Part B: Methodological, 15(2), 105-111.

Huang, S., W. Ren, and S. C. Chan. 2000. "Design and performance evaluation of mixed manual and automated control traffic." IEEE Trans. on Sys., Man, and Cybernetics - Part A: Sys. and Humans, 30(6), 661-673.

ISO. 2010. ISO 15622:2010(en), Intelligent transport systems - Adaptive cruise control systems

- Performance requirements and test procedures. International Organization for Standardization, Geneva, Switzerland.

Jerath, K., and Brennan, S. N. 2012. “Analytical prediction of self-organized traffic jams as a function of increasing ACC penetration." IEEE Trans. on Intel. Transp. Sys., 13(4), 1782-1791.

Kesting, A., Treiber, M., Schönhof, M., and Helbing, D. 2007a. "Extending adaptive cruise control to adaptive driving strategies." Transp. Res. Rec., 2000, 16-24.

Kesting, A., Treiber, M., Schönhof, M., Kranke, F., and Helbing, D. 2007b. "Jam-avoiding adaptive cruise control (ACC) and its impact on traffic dynamics." Traffic and Granular Flow'05, A. Schadschneider, T. Pöschel, R. Kühne, M. Schreckenberg, and D. E. Wolf, eds., Springer, Berlin, Heidelberg, 633-643.

Kesting, A., and M. Treiber. 2008. "Calibrating car-following models by using trajectory data: Methodological study." Transp. Res. Rec., 2088, 148-156.

Kesting, A., M. Treiber, and D. Helbing. 2010. "Enhanced intelligent driver model to access the impact of driving strategies on traffic capacity." Phil. Trans. of the Royal Society A: Mathematical, Physical and Eng. Sciences, 368(1928), 4585-4605.

Lewis, P., and A. Grossman. 2019. Beyond speculation 2.0: An update to Eno's action plan for federal, state, and local policymakers. Eno Center for Transportation, Washington, DC.

Li, Z., W. Li, S. Xu, and Y. Qian. 2015. "Stability analysis of an extended intelligent driver model and its simulations under open boundary condition." Physica A: Statistical Mech. and its Applications, 419, 526-536. 
Liebner, M., M. Baumann, F. Klanner, and C. Stiller. 2012. "Driver intent inference at urban intersections using the intelligent driver model.” 2012 IEEE Intel. Veh. Symposium, 1162-1167.

Mahmassani, H. S., Elfar, A., Shladover, S., and Huang, Z. 2018. Development of an analysis/modeling/simulation (AMS) framework for V2I and connected/automated vehicle environment. Federal Highway Administration, Washington, DC, 180.

Makridis, M., Mattas, K., and Ciuffo, B. 2020. "Response time and time headway of an adaptive cruise control: an empirical characterization and potential impacts on road capacity." IEEE Trans. on Intel. Transp. Sys., 21(4), 1677-1686.

Milanés, V., and S. E. Shladover. 2014. "Modeling cooperative and autonomous adaptive cruise control dynamic responses using experimental data.” Transp. Res. Part C: Emerging Tech., 48(0).

Miller, J. S., and D. Kang. 2019. Ways to consider driverless vehicles in Virginia long-range travel demand models. Virginia Transportation Research Council, Charlottesville, VA.

National Conference of State Legislatures. 2019. "Autonomous vehicles | Self-driving vehicles enacted legislation." National Conference of State Legislatures. Accessed July 10, 2019. http://www.ncsl.org/research/transportation/autonomous-vehicles-self-driving-vehiclesenacted-legislation.aspx.

Ntousakis, I. A., I. K. Nikolos, and M. Papageorgiou. 2015. "On microscopic modelling of adaptive cruise control systems." Transp. Res. Procedia, 4th International Symposium of Transport Simulation (ISTS'14) Selected Proceedings, Ajaccio, France, 1-4 June 2014, 6, 111-127.

Olstam, J. J., A. and Tapani. 2004. Comparison of car-following models. Swedish National Road and Transport Research Institute, Linkping, Sweden.

PTV AG. (2018). PTV Vissim 10 user manual. PTV Group, Karlsruhe, Germany, 1155.

Shladover, S. E., Su, D., and Lu, X.-Y. 2012. "Impacts of cooperative adaptive cruise control on freeway traffic flow." Transp. Res. Rec., 2324, 63-70.

Su, P. P., J. Ma, T. W. P. Lochrane, D. J. Dailey, and D. Hale. 2016. "Integrated adaptive cruise control car-following model based on trajectory data." 95th Annual Meeting of the Transp. Res. Board, Washington, DC.

Sukennik, P. 2018. Micro-simulation guide for automated vehicles. European Union's Horizon 2020 Research and Innovation Programme.

Transportation Research Board. 2010. Highway capacity manual 2010. Transportation Research Board, Washington D.C.

Treiber, M., Hennecke, A., and Helbing, D. 2000. "Congested traffic states in empirical observations and microscopic simulations.” Physical Review E, 62(2), 1805-1824.

VanderWerf, J., Shladover, S. E., Miller, M. A., and Kourjanskaia, N. 2002. "Effects of adaptive cruise control systems on highway traffic flow capacity." Transp. Res. Rec., 1800, 78-84.

VanderWerf, J., Shladover, S., Kourjanskaia, N., Miller, M., and Krishnan, H. 2001. "Modeling effects of driver control assistance systems on traffic:" Transp. Res. Rec., 1748, 167-174. 
Wayland, M. 2015. "Adaptive cruise control goes mainstream.” The Detroit News. Accessed July 30, 2019. https://www.detroitnews.com/story/business/autos/2015/03/03/adaptivecruise-control-growing/24352141/.

Weinberger, M., H. Winner, and H. Bubb. 2001. "Adaptive cruise control field operational testThe learning phase." JSAE Review, 22(4), 487-494.

Wiedemann, R. 1974. Simulation des strassenverkehrsflusses. Schriftenreihe des Instituts für Verkehrswesen der Universität Karlsruhe, Karlsruhe, Germany.

Wiedemann, R., and U. Reiter. 1992. The simulation system MISSION, background and actual state. CEC, Brussels.

Xiao, L., and Gao, F. 2010. "A comprehensive review of the development of adaptive cruise control systems.” Veh. Sys. Dynamics, Taylor \& Francis, 48(10), 1167-1192.

Yuan, Y.-M., Jiang, R., Hu, M.-B., Wu, Q.-S., and Wang, R. 2009. "Traffic flow characteristics in a mixed traffic system consisting of ACC vehicles and manual vehicles: A hybrid modelling approach." Physica A: Statistical Mechanics and its Applications, 388(12), 2483-2491. 